\title{
Caractérisation dynamique aux barres de Hopkinson par chargement indirect en traction d'éprouvettes métalliques collées
}

\author{
Grégory Haugou ${ }^{1,2}$, Jacky Fabis $^{1}$ et Pascal Drazetic ${ }^{2, \text { a }}$
}

1 Département de mécanique du solide et de l'endommagement, Office national d'études et de recherches aérospatiales, 5 boulevard Paul Painlevé, 59045 Lille Cedex, France

2 Laboratoire d'automatique, de mécanique et d'informatique industrielles et humaines, (LAMIH/DRC, UMR-CNRS 8530), Université de Valenciennes et du Hainaut-Cambrésis, Le Mont-Houy, Bâtiment Jonas 2, 59313 Valenciennes Cedex 9, France

Reçu le 19 septembre 2003, accepté le 8 juillet 2005

\begin{abstract}
Résumé - L'article propose une configuration de traction directe d'éprouvettes plates par chargement indirect sur un dispositif de barres de Hopkinson. La fixation par collage des éprouvettes permet de limiter la dégradation des ondes incidente et transmise à laquelle conduit généralement la sollicitation de traction, et qui ne permet plus d'identifier la loi de comportement du matériau considéré. L'intérêt de la configuration proposée réside par ailleurs dans la possibilité d'accéder à la phénoménologie de la sollicitation, impossible dans la plupart des solutions existantes. La campagne d'essais illustrée ici a été réalisée aux vitesses de déformation moyennes comprises entre 200 et $450 \mathrm{~s}^{-1}$ pour une nuance métallique réputée sensible à la vitesse de déformation : l'acier doux XES. Une confrontation des relations rationnelles est faite avec des campagnes d'essais entreprises sur vérin hydraulique et barres de Hopkinson en compression afin d'envisager un recouvrement partiel des lois de comportement obtenues aux vitesses de déformation moyennes classiquement inaccessibles.
\end{abstract}

Mots clés : Recouvrement de moyens d'essais dynamiques / vitesses moyennes de déformation / barres de Hopkinson / traction par chargement indirect

\begin{abstract}
Dynamic characterization using Hopkinson bars through non direct tensile loading tests on glued metallic sheet specimens. This paper deals with a configuration for tensile sheet specimens placed on a split Hopkinson bars apparatus. The specimens are secured by an epoxy glue so as to avoid incident and transmitted waves degradation which limit the access to material behaviour laws using this kind of solicitation and do not permit the identification of material behaviour. The interest in this configuration is to have access to solicitation phenomena, generally not possible in most existing solutions. Tensile tests have been conducted at moderate strain rates between 200 and $450 \mathrm{~s}^{-1}$ on a strain rate sensitive metallic alloy, XES steel. A comparison of the true relations is made between tensile tests performed on a hydraulic machine and a modified SHPB to determine a partial overlapping domain for moderate plastic strain rates classically not accessible.
\end{abstract}

Key words: Dynamic facilities overlapping / moderate strain rates / split Hopkinson bars devices / no-direct tensile loading

\section{Introduction}

La connaissance précise des lois de comportement viscoplastiques des matériaux utilisés dans l'industrie des moyens de transport est devenue essentielle afin de répondre au besoin de rationalisation dans le dimensionnement des structures. Pendant longtemps, ce

a Auteur correspondant :

pascal.drazetic@univ-valenciennes.fr dimensionnement était basé sur l'utilisation de lois de comportement élastoplastiques auxquelles était affecté un coefficient de sécurité important imposé pour résister aux sollicitations de type crash et impact [1]. Or, le développement de moyens d'essais de caractérisation pour les sollicitations dynamiques tels les dispositifs par barres de Hopkinson [2], les machines à pendule [3,4], les volants d'inertie [1], les vérins hydrauliques $[5,6]$ ont permis de mener de vastes campagnes de caractérisation sur les 


\section{Nomenclature}

\begin{tabular}{|c|c|}
\hline$C_{\mathrm{BE}}, C_{\mathrm{BS}}, C_{\mathrm{PROJ}}, C_{\mathrm{E}}$ & célérité dans les barres entrante/sortante, le projectile et l'échantillon $\left(\mathrm{m} . \mathrm{s}^{-1}\right)$ \\
\hline$E_{\mathrm{BS}}$ & module d'élasticité de la barre sortante $(\mathrm{MPa})$ \\
\hline$F_{\mathrm{BE}}, F_{\mathrm{BS}}$ & effort aux interfaces des barres entrante/sortante $(\mathrm{N})$ \\
\hline$I_{\mathrm{BE}}, I_{\mathrm{BS}}$ & impédance acoustique des barres entrante et sortante $\left(\mathrm{kg} . \mathrm{s}^{-1}\right)$ \\
\hline$L_{\mathrm{BE}}, L_{\mathrm{BS}}, L_{\mathrm{PROJ}}$ & longueur des barres entrante/sortante et du projectile $(\mathrm{m})$ \\
\hline$L 1$ & longueur utile de l'échantillon (m) \\
\hline$M_{\mathrm{BE}}, M_{\mathrm{PROJ}}$ & masse de la barre entrante et du projectile $(\mathrm{kg})$ \\
\hline$S_{\mathrm{BE}}, S_{\mathrm{BS}}, S_{\mathrm{E}}$ & section des barres entrante/sortante et de l'échantillon $\left(\mathrm{m}^{2}\right)$ \\
\hline$v_{\mathrm{BE}}, v_{\mathrm{PROJ}}$ & vitesse de la barre entrante et du projectile $\left(\mathrm{m} . \mathrm{s}^{-1}\right)$ \\
\hline$v_{\mathrm{R}}, v_{\mathrm{P}}$ & vitesse de rebond du projectile et vitesse particulaire $\left(\mathrm{m} . \mathrm{s}^{-1}\right)$ \\
\hline$\varepsilon_{\mathrm{E}}$ & déformation plastique conventionnelle de l'échantillon (.) \\
\hline$\varepsilon_{\mathrm{I}}, \varepsilon_{\mathrm{R}}, \varepsilon_{\mathrm{T}}$ & déformation élastique des ondes incidente, réfléchie et transmise (.) \\
\hline$\dot{\varepsilon}_{\mathrm{E}}$ & vitesse de déformation plastique de l'échantillon $\left(\mathrm{s}^{-1}\right)$ \\
\hline$\lambda$ & longueur de l'onde incidente (m) \\
\hline$\rho_{\mathrm{BE}}, \rho_{\mathrm{BS}}, \rho_{\mathrm{E}}$ & masse volumique des barres entrante/sortante et de l'échantillon $\left(\mathrm{kg} \cdot \mathrm{m}^{-3}\right)$ \\
\hline$\sigma_{\mathrm{E}}$ & contrainte plastique conventionnelle de l'échantillon (MPa) \\
\hline$\tau$ & durée du palier élastique (s) \\
\hline
\end{tabular}

matériaux usuellement utilisés [7,8], et ainsi observer l'effet dynamique sur leurs propriétés mécaniques. Ainsi, la sensibilité de certaines nuances métalliques à la vitesse de sollicitation a pu être mise en évidence sur une vaste plage de vitesses de déformation, c'est-à-dire depuis les sollicitations quasi-statiques jusque quelques $10^{3} \mathrm{~s}^{-1}$. Or, un domaine apparaît aux limites d'exploitation généralement admises par les utilisateurs de machines hydrauliques et de barres de Hopkinson. Pour les vérins hydrauliques, les limites sont inhérentes aux problèmes de résonance mécanique auxquels sont sensibles les montages d'essais équipés de cellule d'effort piézoélectrique [5, 6,9-12]. Les limites des dispositifs classiques de mesure par barres de Hopkinson $[7,13,14]$ constituent la borne inférieure de ce domaine; ici, les lois obtenues restent partielles en terme de déformation plastique pour les vitesses de déformation moyennes. Or, ces deux limites technologiques occultent partiellement un domaine essentiel de vitesses de déformation observables localement lors des crashs de véhicules de transport, soit le domaine [1; $5000 \mathrm{~s} \mathrm{~s}^{-1}$. La présence d'un domaine mal connu pose le problème du crédit à accorder aux moyens d'essais sur la validité des lois de comportement matérielles obtenues, et met en exergue le sens physique à donner à une interpolation des lois de comportement vers le domaine des hautes ou des basses vitesses de déformation. Dans le cadre de l'article proposé, les capacités de recouvrement partiel des moyens d'essais sont vérifiées pour un dispositif de traction direct par chargement indirect par barres de Hopkinson et une machine hydraulique rapide. La comparaison des résultats de chacun de ces deux moyens permet de valider la configuration proposée ici sur un dispositif classique de barres de Hopkinson, et de vérifier le recouvrement des lois de comportement du matériau considéré aux vitesses moyennes de déformation.

\section{Relations théoriques}

Les relations de la théorie de propagation des ondes élastiques dans un milieu fini $[15,16]$ permettent aisément de vérifier un certain nombre de limitations auxquelles conduit l'utilisation des barres de Hopkinson. L'équation du mouvement le long de barres cylindriques (Éq. (1)) permet d'établir des relations récurrentes dans la littérature qui ne sont pas rappelées ici

$$
\frac{\partial \sigma}{\partial x}=\rho \times \frac{\partial^{2} u}{\partial t^{2}}
$$

Les relations de base utilisées pour déterminer - à vitesse de déformation constante - la loi de comportement sont rappelées dans les équations (2) et (3) :

$$
\begin{aligned}
& \varepsilon_{\mathrm{R}}(t)=\frac{\dot{\varepsilon}_{\mathrm{E}}(t) \cdot L 1}{2 \cdot C_{\mathrm{BE}}} \\
& \varepsilon_{\mathrm{T}}(t)=\frac{S_{\mathrm{E}} \cdot \sigma_{\mathrm{E}}(t)}{S_{\mathrm{BS}} \cdot E_{\mathrm{BS}}}
\end{aligned}
$$

Un raisonnement basé sur l'égalité de l'énergie fournie par le projectile puis transmise à la barre entrante (Éq. (4)) permet de quantifier - a priori - la vitesse du projectile et sa vitesse de rebond, si celle-ci existe :

$$
\frac{1}{2} \cdot M_{\mathrm{PROJ}} \cdot\left(v_{\mathrm{PROJ}}^{2}-v_{\mathrm{R}}^{2}\right)=\frac{1}{2} \cdot M_{\mathrm{BE}} \cdot v_{\mathrm{BE}}^{2}
$$

La vitesse du projectile est alors donnée par l'équation (5)

$$
v_{\mathrm{PROJ}}=v_{\mathrm{BE}} \cdot \frac{\left(I_{\mathrm{PROJ}}+I_{\mathrm{BE}}\right)}{2} \cdot \sqrt{\frac{M_{\mathrm{BE}}}{M_{\mathrm{PROJ}} \cdot I_{\mathrm{PROJ}} \cdot I_{\mathrm{BE}}}}
$$


L'expression de l'impédance acoustique est formulée à travers l'équation (6) :

$$
I_{\mathrm{O}}=C_{0} \times \rho \times S_{0}
$$

où $C_{0}$ est la célérité du son dans le matériau [17], $\rho$ la masse volumique des barres et $S_{0}$ la section du milieu dans lequel se déplace l'onde élastique.

L'équation (7) permet de déduire la vitesse particulaire dans la barre entrante :

$$
v_{\mathrm{P}}=\frac{I_{\mathrm{PROJ}}}{I_{\mathrm{PROJ}}+I_{\mathrm{BE}}} \cdot v_{\mathrm{PROJ}}
$$

La vitesse de rebond du projectile s'exprime alors selon la relation (8) :

$$
v_{\mathrm{R}}=2 \cdot v_{\mathrm{P}}-v_{\mathrm{PROJ}}
$$

Il est alors montré que la vitesse de rebond du projectile prend une valeur nulle sous condition que les impédances du projectile et de la barre entrante soient égales (Éq. (6)), dans ce cas $v_{\mathrm{P}}=0,5 . v_{\mathrm{PROJ}}$. Il est par ailleurs recommandé que les diamètres et les matériaux des barres de mesure et du projectile soient identiques $[18,19]$ afin de transmettre complètement l'énergie du projectile à la barre entrante, et donc éviter le rebond du projectile. Dans le cas contraire, une correction de l'amplitude des ondes élastiques est nécessaire. Une connaissance précise des célérités et des masses volumiques des barres est requise afin de calculer les états de contrainte, de déformation et de vitesse de déformation subis au cours du temps au sein de l'échantillon. Le module d'élasticité moyen a été calculé par le biais de l'équation (9)

$$
C=\sqrt{\frac{E}{\rho}}
$$

Sous sollicitation de traction/compression, l'état de contrainte dans l'échantillon est uniaxial et les déformations plastiques apparaissent dans les trois directions (Éqs. (10) et (11))

$$
\begin{aligned}
\sigma & =\left|\begin{array}{ccc}
\sigma_{1} & 0 & 0 \\
0 & 0 & 0 \\
0 & 0 & 0
\end{array}\right| \\
\varepsilon & =\left|\begin{array}{ccc}
\varepsilon_{1} & 0 & 0 \\
0 & \varepsilon_{2} & 0 \\
0 & 0 & \varepsilon_{3}
\end{array}\right|
\end{aligned}
$$

\section{Chargement indirect par barres de Hopkinson}

Le dispositif - schématisé à la figure 1 - est constitué de deux barres en Marval 18 (Wt\% 0,01C-0,02Si$0,02 \mathrm{Mn}-18,4 \mathrm{Ni}-4,8 \mathrm{Mo}-0,1 \mathrm{Al}-8,2 \mathrm{Co}-0,45 \mathrm{Ti}-0,028 \mathrm{Br})$ dont la limite élastique est portée à $1700 \mathrm{MPa}$. Les moyens de mesure requis sont essentiellement des jauges

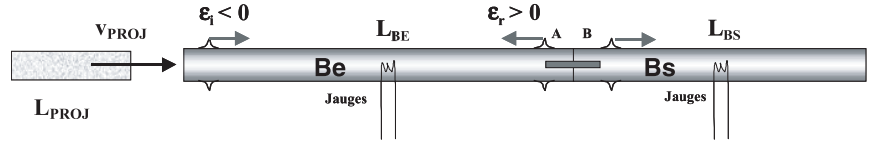

Fig. 1. Schéma de principe de la sollicitation par barres de Hopkinson.

Diagramme Lagrangien de traction indirecte

$\begin{array}{llllllllll}-1100 & -300 & 500 & 1300 & 2100 & 2900 & 3700 & 4500 & 5300 & 6100\end{array}$

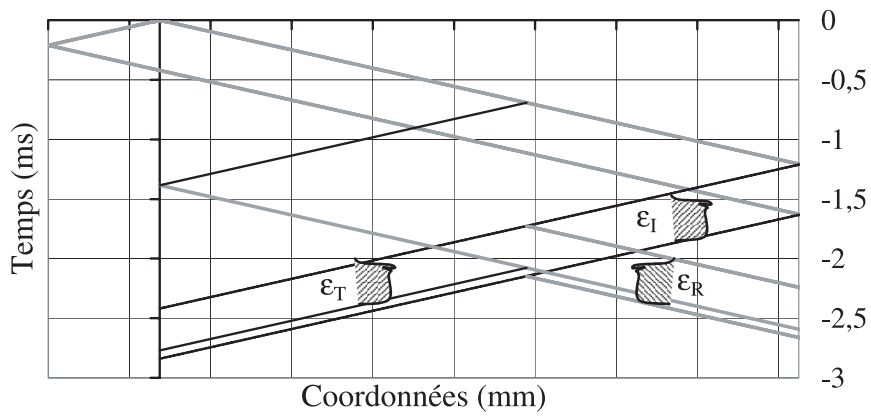

Fig. 2. Diagramme lagrangien de la configuration de traction indirecte.

de déformation de type CEA-06-125-UN-350 (Réf. Measurements group) montées en pont complet. La longueur de grille est de $3,2 \mathrm{~mm}$, et permet l'accès à une bande de fréquence largement supérieure à celle couverte lors d'une sollicitation de type impact, soit $50 \mathrm{kHz}$. Les jauges sont disposées au milieu de chacune des barres, deux en vis-àvis dans le sens longitudinal, deux autres radialement afin de compenser les effets de flexion et intégrer le caractère tridimensionnel des ondes élastiques (non négligeable pour les barres de diamètre élevé). Le conditionnement des jauges de déformation est assuré au moyen d'un ampli-conditionneur Vishay-Micromesures 2310. L'acquisition des données est assurée au moyen d'un enregistreur de type Nicolet MultiPro possédant 8 voies d'acquisition à une fréquence d'échantillonnage de $1 \mathrm{Mech} . \mathrm{s}^{-1}$.

La longueur des barres de mesure et la position des ponts de jauges sur la barre sortante ont été déterminées au moyen d'un diagramme lagrangien [20] afin d'assurer l'absence d'une superposition des systèmes d'ondes élastiques (Fig. 2).

L'onde incidente $\mathrm{C} 1$, qui nait lors du contact dynamique du projectile avec la barre entrante, comprime celle-ci sur une longueur d'onde $\lambda$ se déplaçant à une célérité $C_{\mathrm{BE}}$. À l'interface de la barre sortante en contact avec la barre entrante, l'énergie apportée par la barre entrante y est complètement déposée. Une onde élastique C2 de même nature se déplace alors dans la barre sortante jusqu'à atteindre la face libre et parcourir dans l'autre sens la même barre. Lors de la réflexion sur la face libre de la barre sortante, l'onde originelle inverse son état de contrainte élastique [18] sur la même longueur $\lambda$ tout en se déplaçant à la célérité $C_{\mathrm{BS}}$; cette onde élastique est nommée C3 (Fig. 3). Seule la barre sortante subit alors 
Signaux bruts d'un essai de traction indirecte sur éprouvettes plates en acier XES

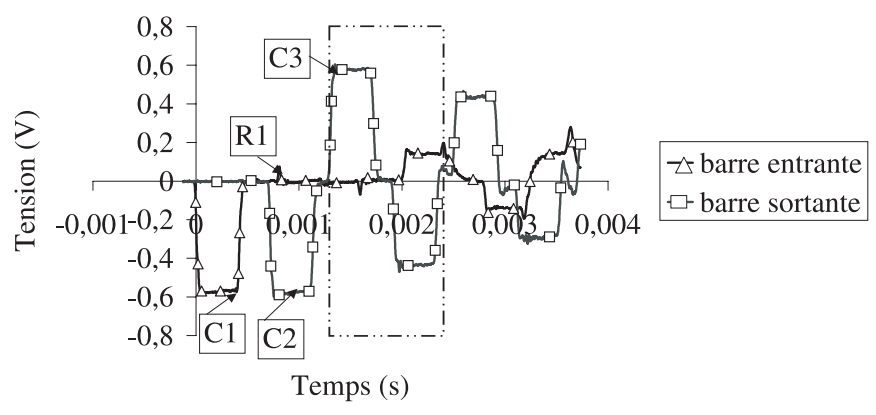

Fig. 3. Illustration des signaux bruts de traction indirecte sur éprouvettes plates XES.

un incrément de déplacement $\delta_{\mathrm{BS}}$ (Éq. (12)), la barre entrante demeurant immobile

$$
\delta_{\mathrm{BS}}=\frac{\mathrm{d}\left(\varepsilon_{\mathrm{I}}(t)+\varepsilon_{\mathrm{R}}(t)\right)}{\mathrm{d} t} \times C_{\mathrm{BS}}
$$

En ce lieu où les ondes de déformation élastique $\varepsilon_{\mathrm{R}}$ et $\varepsilon_{\mathrm{T}}$ s'opposent en amplitude, l'éprouvette subit un chargement de traction pendant la durée $\tau$. Le taux de déformation de l'éprouvette est directement proportionnel à la vitesse de la barre sortante, et donc du projectile (Éqs. (2), (8), (12)). L'ensemble des trois ondes illustrées dans l'encadré de la figure 3 est alors directement exploitable pour identifier la loi de comportement du jeu d'éprouvettes considéré sur la base des relations exposées dans le paragraphe précédent.

Un projectile de longueur 1,1 m a été retenu afin d'obtenir des paliers de déformation élastique de l'ordre de $420 \mu$ s environ. Cette durée permet d'accéder à des taux de déformation plastique de matériaux métalliques ductiles suffisants. La barre sortante a une longueur de 2,7 m. Les faces de chacune des barres ont été dressées pour assurer un temps de montée rapide du front élastique; celui-ci est de l'ordre de $40 \mu \mathrm{s}$. La barre entrante a une longueur de 3,6 m permettant d'éviter une éventuelle superposition d'une onde initialement réfléchie avec l'onde transmise à la barre entrante.

Des essais à vide de barres jointives ont été préalablement réalisés pour déterminer les célérités de chacune des barres de mesure (Tab. 1). Ce type d'essais permet de vérifier la qualité des ondes élastiques, et notamment la rapidité du temps de montée de l'onde incidente. Les caractéristiques mécaniques et physiques obtenues pour les barres confirment les données usuellement obtenues pour la nuance considérée [21,22].

Le logiciel de dépouillement automatique DAVID [23] a été utilisé pour l'ensemble des résultats présentés ici. Un dépouillement simplifié a été réalisé, celui-ci se basant sur la vérification d'un bon état équilibre des forces aux interfaces des barres de mesure [24].
Tableau 1. Dimensions et caractéristiques des barres de traction par chargement indirect.

\begin{tabular}{cccccc} 
& $L(\mathrm{~m})$ & $\varnothing(\mathrm{mm})$ & $S_{\mathrm{ep}}\left(\mathrm{mm}^{2}\right)$ & $\rho\left(\mathrm{kg} \cdot \mathrm{m}^{-3}\right)$ & $C\left(\mathrm{~m}_{\mathrm{s}} \mathrm{s}^{-1}\right)$ \\
\hline $\begin{array}{c}\text { barre } \\
\text { entrante }\end{array}$ & 3,6 & 25 & 491 & 8200 & 4630 \\
\hline $\begin{array}{c}\text { barre } \\
\text { sortante }\end{array}$ & 2,7 & 25 & 491 & 8170 & 4600 \\
\hline & & & & & \\
\hline
\end{tabular}

Fig. 4. Éprouvettes plates de traction - Dimensions exprimées en $\mathrm{mm}$.

\section{Préparation des éprouvettes plates métalliques}

La vitesse maximale du projectile est fixée à $10 \mathrm{~m} . \mathrm{s}^{-1}$ en raison des risques de décollement des jauges de déformation. Une vitesse minimale de $3 \mathrm{~m} . \mathrm{s}^{-1}$ est imposée par la limitation du système de lancement des projectiles longs. Les éprouvettes en acier doux XES ( $W t \%$ $\mathrm{C}=0,05-\mathrm{Cr}<0,01-\mathrm{Cu}=0,013-\mathrm{Mn}=0,018-\mathrm{Al}=$ $0,064-\mathrm{Ni}=0,02)$ sont prélevées dans le sens de laminage de tôles d'acier d'épaisseur égale à 1,17 mm. La longueur utile $L 1$ des éprouvettes a été prise égale à 11,3 mm afin de s'assurer de vitesses de déformation plastique proches de celles convoitées initialement pour établir le recouvrement (Fig. 4).

Le choix du principe s'appuie sur un objectif majeur : celui de minimiser la rupture d'impédance à laquelle conduit généralement la configuration de traction [24-27]. Toute discontinuité importante apparaissant le long des barres devient alors pénalisante [28]. Or, les modifications de mise en place de l'échantillon pour sa caractérisation en traction sont liées au maintien d'une partie non déformable de l'éprouvette sur chaque barre. Généralement, cette contrainte de positionnement, par filetage ou goupillage, de l'éprouvette conduit à une rupture d'impédance apportée par les changements de section dans les barres [29-31] ou dans les montages [8,26,32].

Une estimation des amplitudes de contrainte $\Gamma_{\mathrm{T}}$ et $\Gamma_{\mathrm{R}}$ dans les barres entrante et sortante est par ailleurs nécessaire afin d'assurer la présence d'un signal transmis d'amplitude suffisante $[15,33]$ à travers la continuité des efforts et des vitesses aux interfaces des barres entrante et sortante (Éqs. (13) et (14)) :

$$
\begin{aligned}
& \Gamma_{\mathrm{R}}=-\frac{\left(S_{\mathrm{BE}} \rho_{\mathrm{BE}} C_{\mathrm{BE}}\right)-\left(S_{\mathrm{E}} \rho_{\mathrm{E}} C_{\mathrm{E}}\right)}{\left(S_{\mathrm{BE}} \rho_{\mathrm{BE}} C_{\mathrm{BE}}\right)+\left(S_{\mathrm{E}} \rho_{\mathrm{E}} C_{\mathrm{E}}\right)} \\
& \Gamma_{\mathrm{T}}=1+\Gamma_{\mathrm{R}}
\end{aligned}
$$


Evolution du coefficient de transmission $\Gamma_{\mathrm{T}}$ en fonction des sections de barres et de l'échantillon

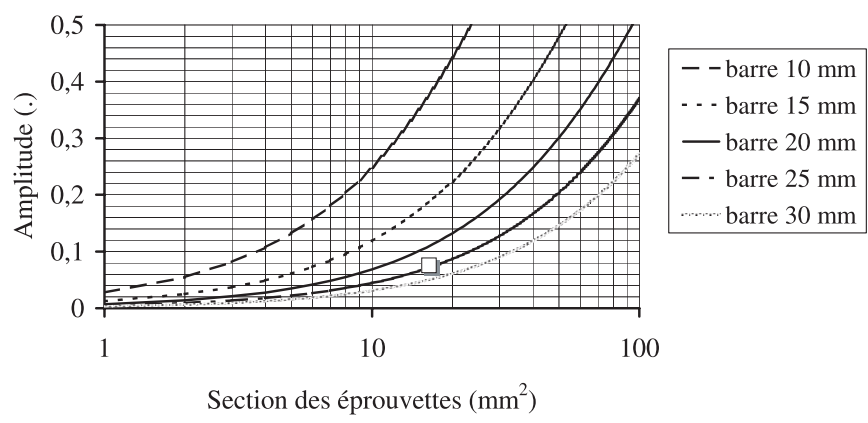

Fig. 5. Évolution des amplitudes des ondes transmise et réfléchie en fonction de la section utile des éprouvettes.

$\Gamma_{\mathrm{T}}$ demeure relativement constante sur la plage de vitesses de déformation. $\Gamma_{\mathrm{T}}$ et $\Gamma_{\mathrm{R}}$ doivent être équitablement réparties, et impliquent des modifications de section sur de grandes variations de vitesses de déformation [15]. Le rapport minimal est de l'ordre de 1 selon les équations (2) et (3). Ainsi, la section utile totale est proche de $20 \mathrm{~mm}^{2}$ pour le dispositif considéré. L'amplification du pont de jauges de la barre véhiculant l'onde transmise peut donc être limitée, et par suite celle des bruits électriques environnants. L'évolution des amplitudes des ondes élastiques est présentée à la figure 5 .

Dans la configuration d'essais proposée, la perte de section dans les manchons est estimée à environ $2 \%$ comparativement à leur section initiale. Cette perte est liée à la présence de logements pour accueillir les éprouvettes. La longueur utile $L 1$ des éprouvettes peut être comprise entre 6,8 et $25 \mathrm{~mm}$ et la largeur est tolérancée pour leur mise en position (Fig. 6). La tenue de la colle au cisaillement étant de $23 \mathrm{MPa}$ après polymérisation, une section minimale de collage d'environ $172 \mathrm{~mm}^{2}$ par éprouvette est nécessaire. Une longueur de collage de $20 \mathrm{~mm}$ sur une largeur de 8,6 mm est donc requise pour assurer la tenue de la colle (Fig. 6). Au terme de l'essai, les manchons sont démontés puis placés dans un bain de solvant (corclène).

Le principe du montage des éprouvettes dans les manchons se décline selon les figures 7 et 8 . Un ponçage et un nettoyage des faces de chaque éprouvette sont nécessaires avant leur mise en place sur les manchons.

Un jeu de 4 éprouvettes plates de section totale utile proche de $20 \mathrm{~mm}^{2}$ est alors disposée dans les logements placés en vis-à-vis sur le pourtour des manchons cylindriques, de même nuance que les barres de mesure. Cette précaution assure la symétrie du chargement. Les éprouvettes sont finalement fixées au moyen d'une colle époxy bicomposants 2014 (Réf. 3M).

Les éprouvettes, placées en leur milieu à l'interface des deux pièces du montage, sont maintenues par une pièce de centrage afin d'assurer leur positionnement (Fig. 8). L'ensemble est ensuite pré-contraint aux extrémités afin d'annuler les jeux éventuels entre les deux manchons qui peuvent demeurer sur la plupart des montages d'éprouvettes plates, notamment à travers l'hyperstaticité liée au goupillage [26].

La polymérisation de la colle n'est effective qu'après plusieurs heures à température ambiante; au terme desquelles aucun jeu résiduel n'apparaît. Passé ce délai, l'ensemble peut alors être inséré à l'interface des deux barres au moyen de bagues filetées. Un serrage conséquent est alors indispensable pour palier tout risque de desserrage des pièces (Fig. 9). Les éprouvettes ne sont ainsi soumises à aucun chargement initial susceptible de les déformer plastiquement lorsque la barre entrante dépose son énergie dans la barre sortante sous la forme d'une onde de compression.

D'autres auteurs se sont attachés à développer une approche similaire par un chargement direct de la barre entrante. Un jeu de logements cylindriques rainurés contenant une éprouvette plate collée peut alors être inséré par filetage [34]. Un goupillage préalable de l'éprouvette permet son positionnement avant collage [35].

\section{Calibration à vide du dispositif d'essais par traction indirecte}

La calibration a pour objectif de quantifier - avant chaque campagne d'essais, et pour une sollicitation donnée - la perte d'énergie liée au transport de l'onde incidente dans la barre sortante; mais également d'évaluer l'influence des montages d'éprouvettes sur la qualité des ondes élastiques. Un coefficient de calibration par vitesse est déterminé par le logiciel de dépouillement automatique DAVID [21] et correspond à la correction à apporter sur l'amplitude de l'onde incidente lors de son transport dans la barre sortante. Le coefficient a été déterminé à partir d'un essai sur barres jointives à $5,2 \mathrm{~m} . \mathrm{s}^{-1}$ et a été évalué à 1,03 (et vaut 1,01 lors de la présence des manchons). L'influence des manchons sur l'allure de l'onde transmise apparaît comme donc négligeable.

La section du projectile et celle de la barre entrante étant différentes, un second coefficient doit pondérer l'amplitude du signal de l'onde incidente afin de compenser le manque d'énergie apportée à la barre entrante; ce coefficient vaut 0,9898 selon l'équation (9). Comparativement à certains montages présentés dans la littérature, l'incidence de la géométrie du montage d'éprouvettes plates reste faible (Fig. 10). Aucune perturbation n'altère l'onde incidente lorsque celle-ci parcourt le montage d'éprouvettes plates. Seule une partie réfléchie de l'onde incidente dans la barre entrante est d'amplitude sensiblement plus élevée. Cette réflexion est liée à un défaut d'alignement des barres de mesure; par la suite elle est amplifiée par la présence des filetages dans le montage. Le décalage temporel des ondes transmises est lié au temps nécessaire pour parcourir les manchons; soit $124 \mathrm{~mm}$ avec une célérité de $4630 \mathrm{~m} . \mathrm{s}^{-1}$. 


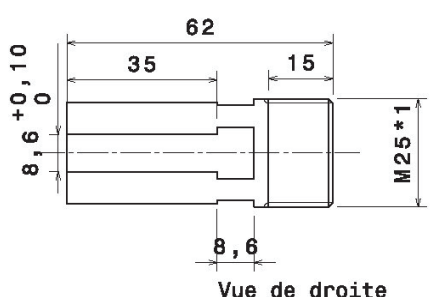

Vue de droite

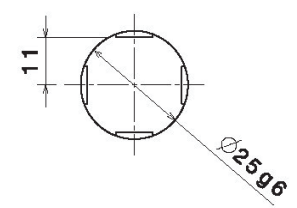

Vue de face

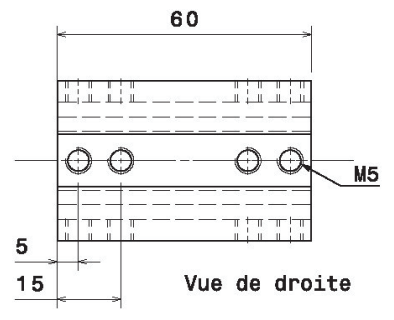

$15 . \Rightarrow \quad$ Vue de droite

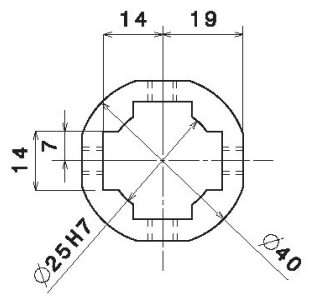

Vue de face

Fig. 6. Montage d'éprouvettes plates par collage sur barres de Hopkinson.
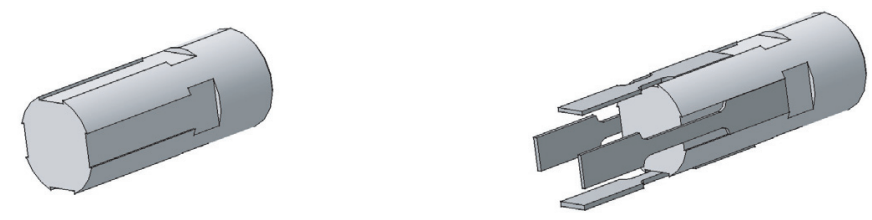

Fig. 7. Mise en place des éprouvettes dans le premier manchon.
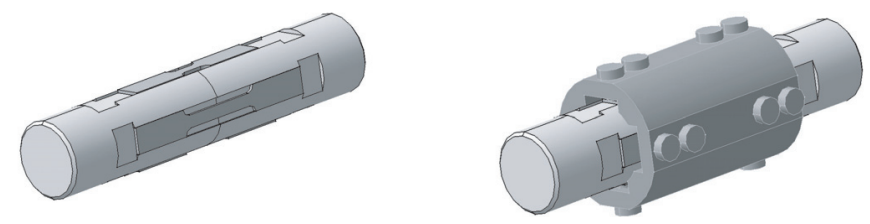

Fig. 8. Mise en place du second manchon pour centrage avant le collage.

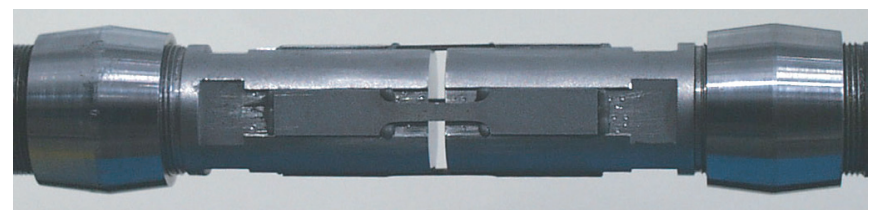

Fig. 9. Ensemble d'éprouvettes collées vissé sur les barres de Hopkinson.

\section{Caractérisation aux barres de Hopkinson de l'acier doux XES}

Une caractérisation de traction dynamique a été envisagée pour un matériau métallique réputé sensible à la vitesse de déformation : l'acier doux XES. Cette sensibilité avérée permet de comparer à différentes vitesses de déformation les lois de comportement obtenues sur différents dispositifs d'essais dynamiques. La mesure de la masse est réalisée au moyen d'une balance précise au $\mu \mathrm{g}$ pour le calcul de la masse volumique du matériau. Le module d'élasticité, peu sensible à la vitesse de déformation, est préalablement obtenu à partir d'essais statique et dynamique menés sur vérin hydraulique rapide. La célérité des ondes dans l'acier XES a alors pour valeur $5325 \mathrm{~m} . \mathrm{s}^{-1}$. La figure 11 illustre les lois matérielles de l'acier considéré aux vitesses de déformation quasi-statique et dynamiques (200 et $\left.440 \mathrm{~s}^{-1}\right)$. Une identification de la loi de comportement est faite sous DAVID, et un lissage est obtenu au

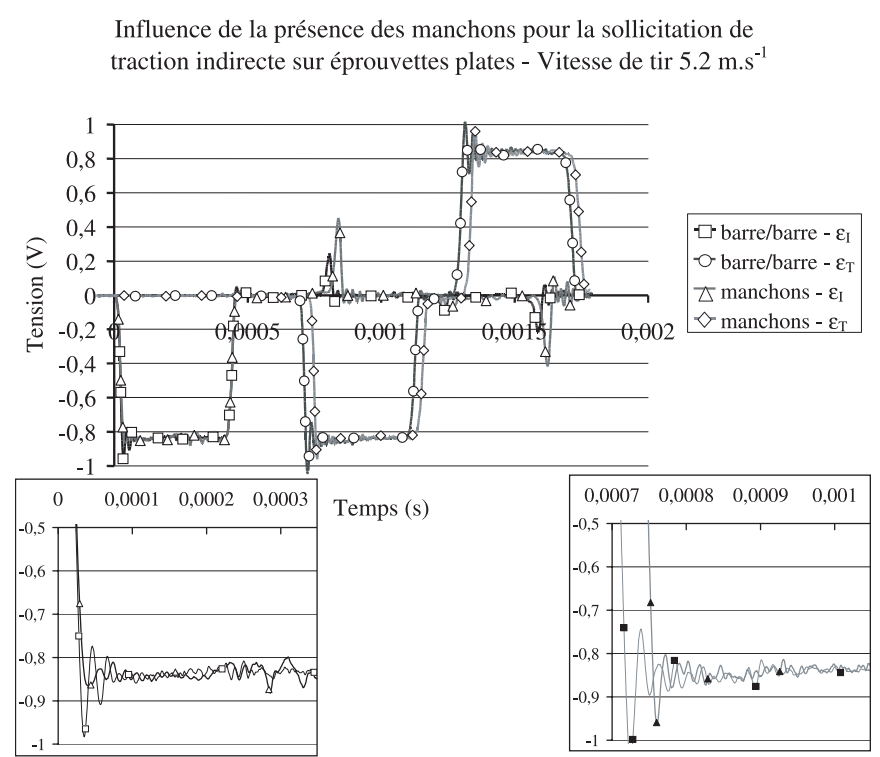

Fig. 10. Influence des manchons sur le transport de l'onde incidente.

Évolution du comportement en traction dynamique d'éprouvettes plates en acier XES - Données brutes

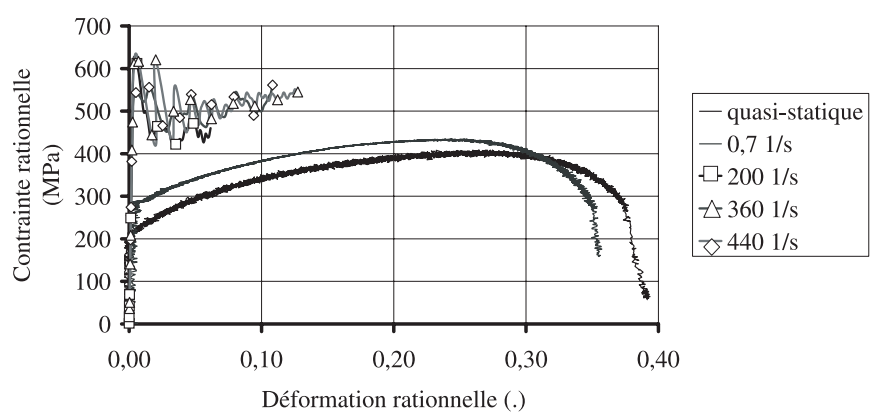

Fig. 11. Relations rationnelles brutes de la nuance d'acier XES.

moyen d'un polynôme de degré $n$ afin d'extraire les oscillations perturbatrices (Fig. 12). Une bonne concordance des courbes rationnelles brutes et traitées est constatée, ce qui leur permet d'être considérées comme exploitables. Les présents essais sont nommés par la suite «SHBDRCS » à titre de comparaison aux essais réalisés par d'autres dispositifs à des vitesses de déformation voisines. 
Évolution du comportement en traction dynamique d'éprouvettes plates en acier XES - Données filtrées

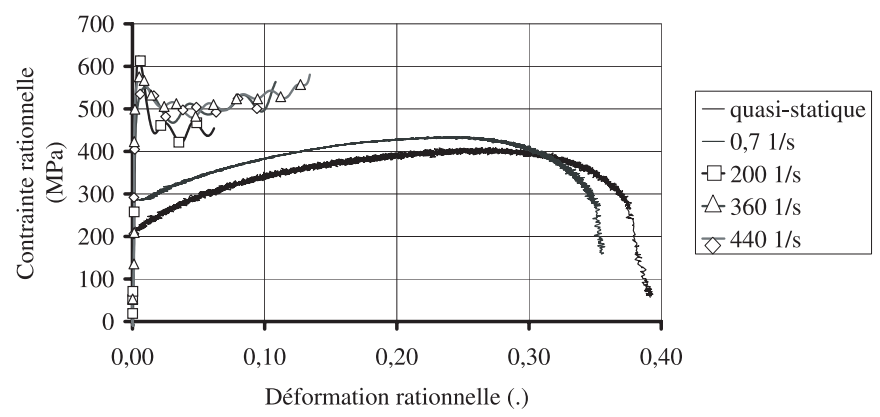

Fig. 12. Relations rationnelles filtrées de la nuance d'acier XES.

Etat d'équilibre d'un chargement de traction indirecte sur éprouvettes plates en acier XES à $200 \mathrm{~s}^{-1}$

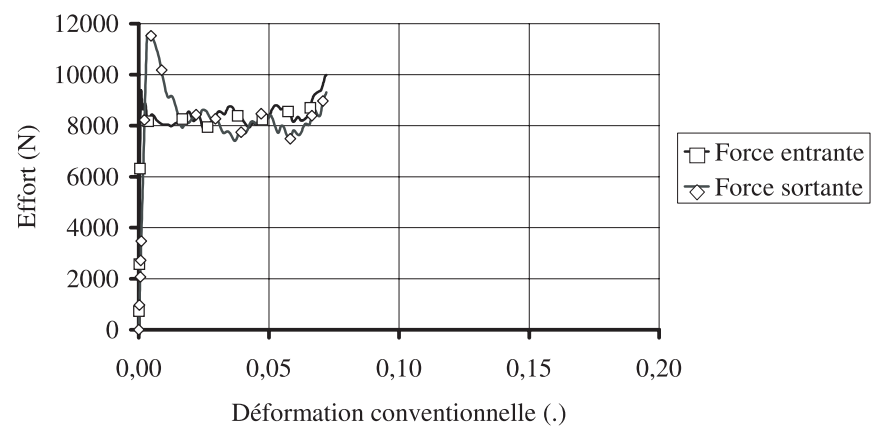

Fig. 13. Évolution de l'équilibre des efforts aux interfaces des barres pour les essais de traction sur l'acier XES - Vitesse de déformation de $200 \mathrm{~s}^{-1}$.

La vérification de l'équilibre des efforts aux interfaces des barres entrante et sortante est indispensable afin de considérer comme valide le comportement obtenu pour l'échantillon placé à leur interface respective. Dans le cas contraire, un déséquilibre des efforts au cours du chargement est significatif d'une dispersion des ondes dans le montage et donc d'un dysfonctionnement dans le mode de chargement de l'échantillon.

Les figures 13 à 15 présentent les évolutions des états de chargement aux faces entrante et sortante du dispositif d'essais lors de la sollicitation en traction des éprouvettes plates en acier XES à 200, 360 et $440 \mathrm{~s}^{-1}$. Les lois de comportement sont déterminées jusque 0,14 de déformation plastique à $440 \mathrm{~s}^{-1}$. Les relations utilisées pour le calcul des efforts aux interfaces sont directement issues de la relation de Hooke (Éqs. (15) et (16)) :

$$
\begin{aligned}
& F_{\mathrm{BE}}=E_{\mathrm{BE}} \cdot S_{\mathrm{BE}} \cdot\left(\varepsilon_{\mathrm{I}}(t)+\varepsilon_{\mathrm{R}}(t)\right) \\
& F_{\mathrm{BS}}=E_{\mathrm{BS}} \cdot S_{\mathrm{BS}} \cdot\left(\varepsilon_{\mathrm{T}}(t)\right)
\end{aligned}
$$

Un bon équilibre du chargement des éprouvettes est vérifié malgré la présence d'oscillations sur la face de la barre entrante dont l'amplitude est perturbante au-delà de $400 \mathrm{~s}^{-1}$.
Etat d'équilibre d'un chargement de traction indirecte sur éprouvettes plates en acier XES à $360 \mathrm{~s}^{-1}$

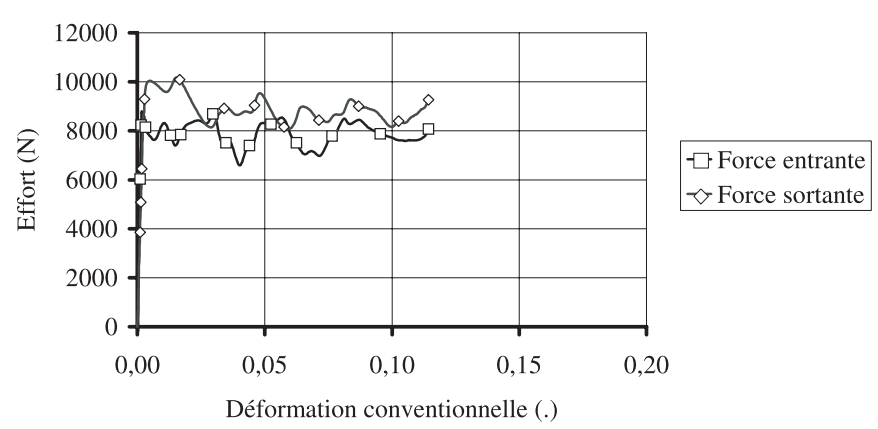

Fig. 14. Évolution de l'équilibre des efforts aux interfaces des barres pour les essais de traction sur l'acier XES - Vitesse de déformation de $360 \mathrm{~s}^{-1}$.

Etat d'équilibre d'un chargement de traction indirecte sur éprouvettes plates en acier XES à $440 \mathrm{~s}^{-1}$

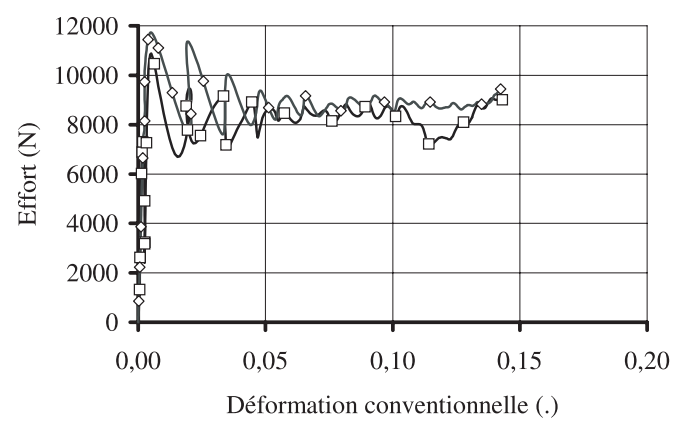

$\square$ Force entrante

Fig. 15. Évolution de l'équilibre des efforts aux interfaces des barres pour les essais de traction sur l'acier XES - Vitesse de déformation de $440 \mathrm{~s}^{-1}$.

La figure 16 présente le contenu fréquentiel des essais dynamiques menés sur éprouvettes plates collées. Sur cette figure sont mises en évidence les oscillations perturbatrices à la vitesse de déformation de $400 \mathrm{~s}^{-1}$. D'un point de vue temporel, la gamme de fréquence [30; 40] kHz correspond aux allers/retours au cours du chargement d'une partie des ondes élastiques dans chacun des manchons filetés. Ces oscillations - initiées par des perturbations dans les filets des manchons - ont pour conséquence de masquer le comportement réel des éprouvettes de caractérisation et impliquent un traitement par des outils mathématiques de lissage.

La figure 17 présente les états de déformation finaux pour les sollicitations de traction sur éprouvettes plates en acier XES à 260 et $380 \mathrm{~s}^{-1}$. La qualité du chargement est vérifiée par le relevé de la cote prise entre les manchons à proximité de chacune des éprouvettes. Un écart de l'ordre du centième de mm a été mesuré entre les manchons après chaque essai. 
Contenu fréquentiel de l'essai de traction indirecte par collage de la nuance d'acier XES

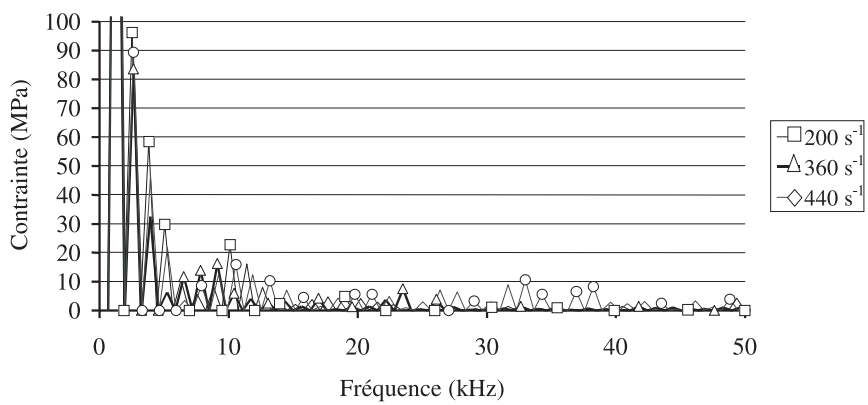

Fig. 16. Contenu fréquentiel des essais dynamiques menés sur la nuance d'acier XES.

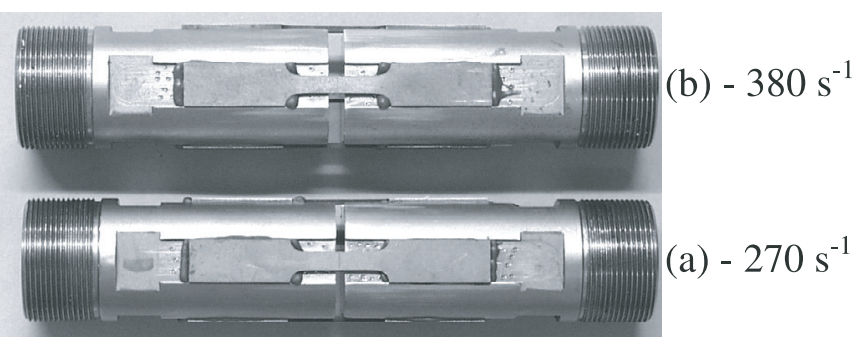

Fig. 17. État de déformation des jeux d'éprouvettes de traction. (a) $260 \mathrm{~s}^{-1}$, (b) $380 \mathrm{~s}^{-1}$. Éprouvettes XES (section totale $18,72 \mathrm{~mm}^{2}$ - Longueur $13 \mathrm{~mm}$ ).

\section{Validation du montage de traction par chargement indirect par comparaison des essais dynamiques}

Deux campagnes d'essais dynamiques entreprises sur le même matériau métallique à des vitesses de déformation plastiques proches ont permis de valider la configuration de traction présentée ici vis-à-vis d'une machine hydraulique et de barres de Hopkinson en traction par compression.

\subsection{Montage de traction d'éprouvettes plates pliées}

La comparaison des résultats obtenus précédemment est faite avec une campagne d'essais de traction dynamique menée au LMS de l'École Polytechnique sur éprouvettes plates pliées [22] (Fig. 18).

Le domaine de vitesses de déformation atteint permet de recouvrir celui des essais de traction sur éprouvettes collées, soit $[160 ; 730] \mathrm{s}^{-1}$. La longueur des parties utiles a été portée à $20 \mathrm{~mm}$ pour une section totale de $36 \mathrm{~mm}^{2}$, l'échantillon possédant 6 zones utiles (Fig. 19). Les éprouvettes ont été prélevées dans la même tôle métallique puis usinées au moyen d'une machine à fil par électroérosion Un ajustement à l'aide de trous de positionnement permet une répétabilité du pliage sous presse quasi-statique. Les essais de cette campagne de caractérisation sont nommés « SHB-LMS ».

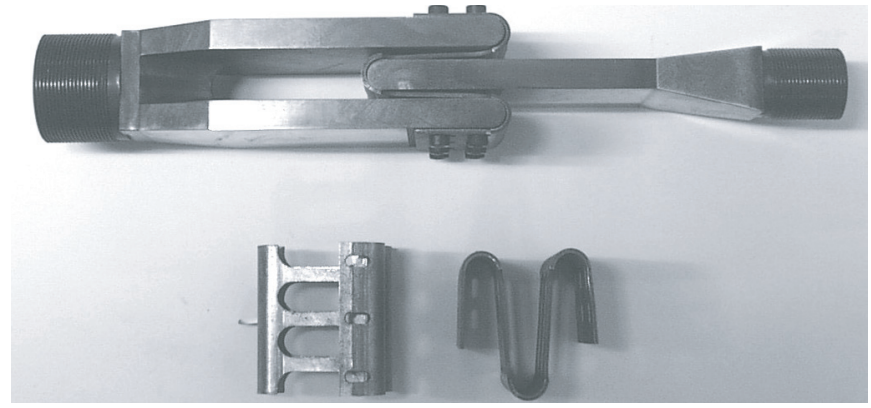

Fig. 18. Montage de traction par compression [22].

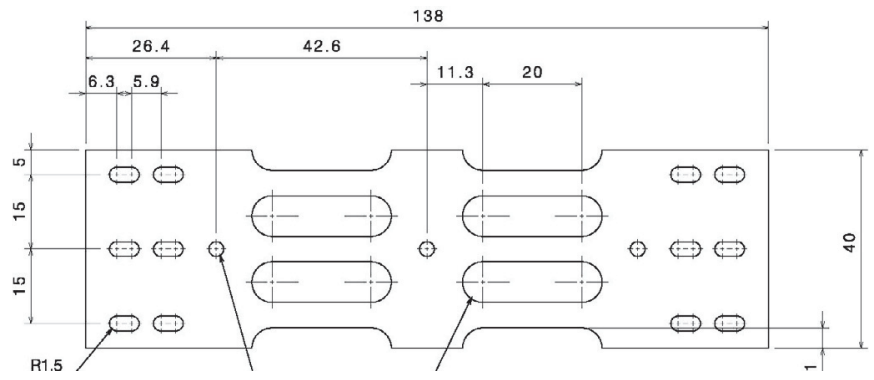

Fig. 19. Géométrie des éprouvettes métalliques plates pliées - Dimensions en mm.

\subsection{Montage d'essais de traction sur vérin hydraulique}

Dans une seconde approche, une campagne d'essais de traction sur une machine hydraulique rapide (VR) asservie en vitesse a été entreprise afin d'établir un domaine de recouvrement en termes de vitesses de déformation pour le domaine $[100 ; 300] \mathrm{s}^{-1}$. Le vérin hydraulique utilisé ici est disponible à l'Onera-Centre de Lille, et peut atteindre une vitesse maximale de $10 \mathrm{~m} . \mathrm{s}^{-1}$. Ses capacités en charge sont $\pm 70 \mathrm{kN}$ en statique et $\pm 50 \mathrm{kN}$ en dynamique pour une course utile de $280 \mathrm{~mm}$. La mesure de l'effort est assurée par une cellule d'effort piézoélectrique de type Kistler 9071A. Des limites maximales en vitesse de déformation sont atteintes en raison d'une résonance mécanique du montage d'essais souvent rappelée dans la littérature [7-9,14,36-38]. Deux géométries d'éprouvettes de traction ont été retenues pour les essais quasi-statiques et dynamiques du matériau métallique considéré afin de vérifier la non-influence de la géométrie d'éprouvettes utilisée sur le dispositif de barres de Hopkinson vis-à-vis de celle utilisée pour les essais de traction sur vérin hydraulique. Des essais de caractérisation quasi-statique de ces deux géométries (Figs. 4 et 20), référencées « type I» et «type II », ont été comparés et ont révélé une bonne concordance des résultats [39].

Pour les essais réalisés sur vérin hydraulique rapide, la longueur utile des éprouvettes de type II est prise égale à $6,8 \mathrm{~mm}$ pour une largeur utile $\mathrm{S} 1 \mathrm{de} 4 \mathrm{~mm}$. La mesure des allongements est réalisée au moyen de l'extensométrie optique (Zimmer $200 \mathrm{XH}$ ), et permet de déterminer les relations conventionnelles des nuances métalliques sous sollicitations quasi-statique et dynamique jusqu'à rupture de l'éprouvette. Les relations perturbées par les oscillations 


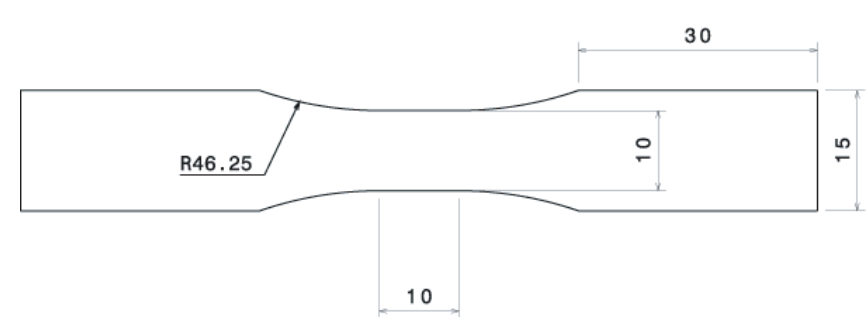

Fig. 20. Géométrie des éprouvettes plates de traction «type $\mathrm{I} »-$ Dimensions en $\mathrm{mm}$.

Comparaison des lois de comportement rationnelles de l'acier XES à une vitesse de déformation de $170 \mathrm{~s}^{-1}$
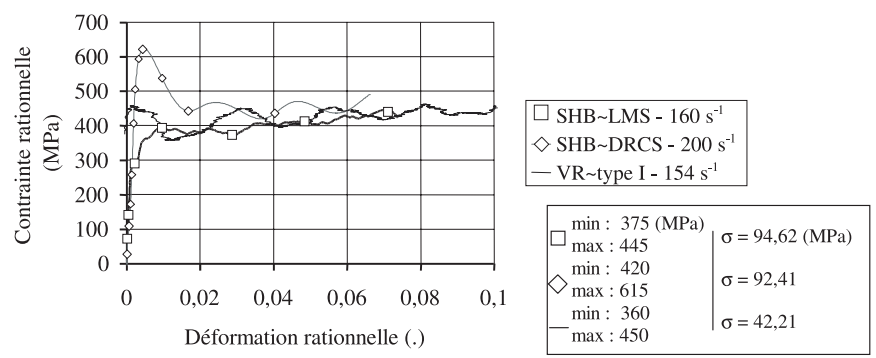

Fig. 21. Comparaison des relations contraintes/déformations rationnelles pour la nuance d'acier XES et une vitesse de déformation moyenne de $170 \mathrm{~s}^{-1}$.

ont été filtrées au moyen d'une analyse par transformation de Fourier pour les fréquences supérieures à $7 \mathrm{kHz}$.

\subsection{Confrontation des résultats d'essais aux vitesses moyennes de déformation}

D'un point de vue qualitatif, les figures 21 à 24 illustrent les lois de comportement rationnelles issues de trois moyens d'essais pour des déformations plastiques de l'acier doux XES allant jusque 0,1 et 0,2 et des vitesses de déformations voisines - la sensibilité de la nuance considérée restant faible sur cette gamme de vitesses de déformation restreinte. Les principaux écarts sont observés dès que la plastification apparaît, puis une stabilisation de la loi d'écrouissage permet de considérer les résultats comme acceptables. Les écarts de contraintes $\sigma$ ainsi que les valeurs de contraintes minimales et maximales sont également illustrés afin de définir un corridor de dispersion. Plusieurs explications permettent d'expliquer les écarts obtenus :

- la présence d'oscillations perturbantes sur la plupart des moyens d'essais,

- les essais sur barres de Hopkinson ne sont pas à vitesse de déformation constante,

- les effets d'inertie provoqués par le chargement indirect de traction.

$\mathrm{Au}$ vu de ces résultats, il paraît alors intéressant de vérifier l'existence d'un domaine partiellement recouvrant en terme de déformation pour la gamme de vitesses de déformation $[160 ; 600] \mathrm{s}^{-1}$ acquis par l'apport de deux moyens d'essais complémentaires.
Comparaison des lois de comportement rationnelles de l'acier XES pour une vitesse de déformation de $240 \mathrm{~s}^{-1}$
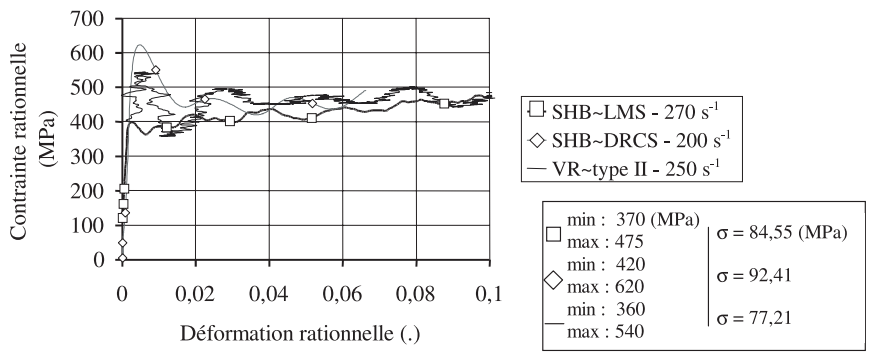

Fig. 22. Comparaison des relations contraintes/déformations conventionnelles pour la nuance d'acier XES et une vitesse de déformation moyenne de $240 \mathrm{~s}^{-1}$.

Comparaison des lois de comportement rationnelles de l'acier XES pour une vitesse de déformation de $350 \mathrm{~s}^{-1}$

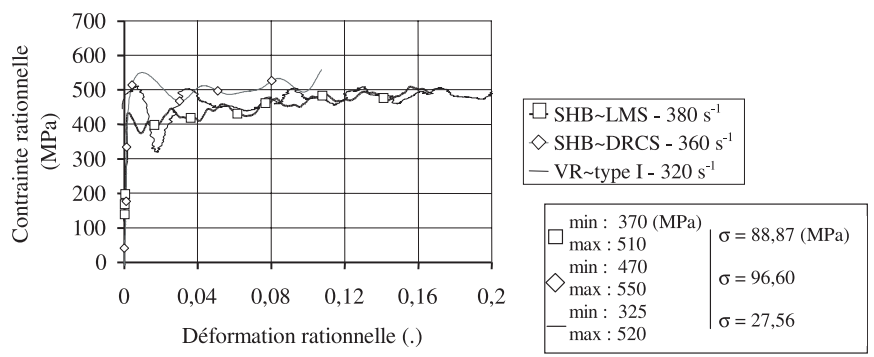

Fig. 23. Comparaison des relations contraintes/déformations conventionnelles pour la nuance d'acier XES et une vitesse de déformation moyenne de $350 \mathrm{~s}^{-1}$.

Comparaison des lois de comportement rationnelles de l'acier XES pour une vitesse de déformation de $520 \mathrm{~s}^{-1}$
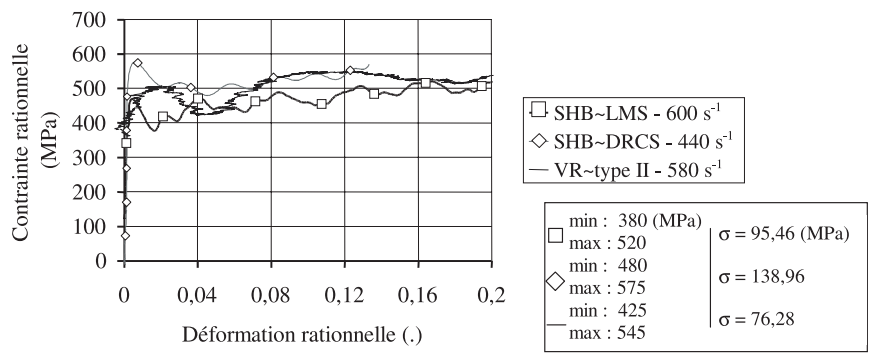

Fig. 24. Comparaison des relations contraintes/déformations conventionnelles pour la nuance d'acier XES et une vitesse de déformation moyenne de $520 \mathrm{~s}^{-1}$.

La figure 25 permet de mettre en exergue l'intérêt du recouvrement des moyens d'essais en terme de vitesses de déformation mais également celui de tendre vers un recouvrement complet du point de vue des déformations plastiques mesurées [39]. Les résultats synthétiques sont présentés pour les essais de traction dynamique sur les éprouvettes plates de type I et II et menés sur vérin hydraulique rapide (VR) et sur deux dispositifs mis en œuvre sur barres de Hopkinson à partir d'éprouvettes plates pliées (SHB LMS) et collées (SHB DRCS). 


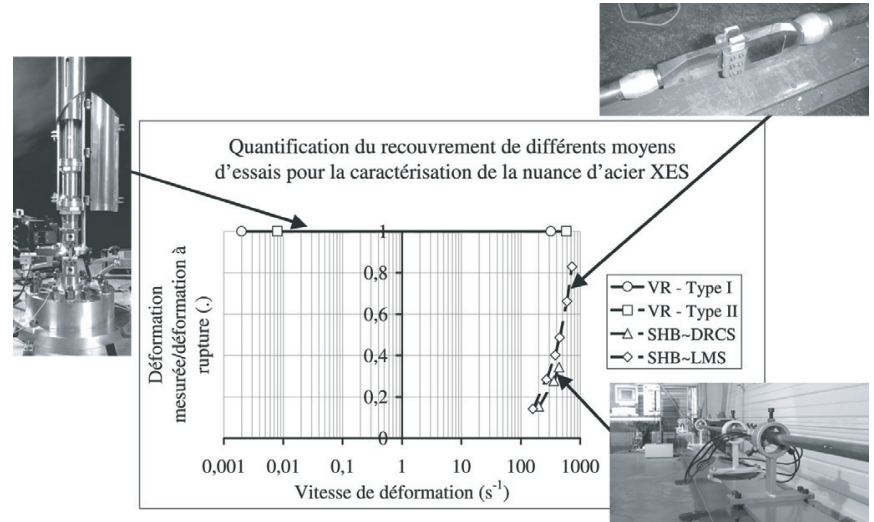

Fig. 25. Domaine de recouvrement partiel établi aux vitesses moyennes de déformation pour la nuance d'acier doux XES.

\section{Conclusions et perspectives}

Un dispositif de traction d'éprouvettes plates par chargement indirect au moyen de barres de Hopkinson a été proposé puis validé. Le choix de la fixation des éprouvettes a été défini par la volonté de réduire au maximum les ruptures d'impédance responsables du comportement incertain de l'échantillon lors de la sollicitation. La configuration proposée repose sur le collage d'éprouvettes plates métalliques (époxy bicomposants 2014) sur le pourtour de manchons cylindriques permettant la visualisation à cadence élevée de la sollicitation des éprouvettes, et laisse entrevoir la mesure locale des déformations plastiques au moyen de l'extensométrie optique. Toutefois, le dispositif par barres de Hopkinson proposé ici reste limité en déformation au sens où les vitesses de déformation inférieures à $100 \mathrm{~s}^{-1}$ ne permettent pas d'identifier une loi matérielle significative. Une longueur de projectile élevée peut alors être envisagée mais impose des barres de longueur d'autant plus conséquente. Trois conséquences directes rendent délicate cette démarche : la mise en vitesse du projectile, les difficultés de guidage et la dispersion des ondes dans des barres très longues. Dans ces conditions, le chargement peut être obtenu par une pré-tension hydraulique d'une partie de la barre entrante. Cette solution permet de supprimer le projectile, l'énergie emmagasinée est ici libérée lors de la rupture du contact permettant la prétension hydraulique. Le traitement des systèmes d'ondes élastiques peut alors être envisagé par des techniques de déconvolution [40,41]. Par ailleurs, la configuration actuelle des manchons cylindriques se limite aux matériaux dont les contraintes ultimes restent de l'ordre de $500 \mathrm{MPa}$. Pour les matériaux à haute limite d'élasticité, une section de collage plus conséquente devient nécessaire $\left(320 \mathrm{~mm}^{2}\right.$ pour des contraintes plastiques de $1000 \mathrm{MPa}$ ).

Une campagne d'essais a été menée sur un matériau métallique sensible à la vitesse de déformation (acier doux XES prélevé dans la tôle originelle selon le sens de laminage convenu initialement) afin de comparer, pour des vitesses de déformation proches, les lois de comportement matérielles rationnelles obtenues sur chaque dispositif d'essais (vérin rapide de l'Onera-Centre de Lille [0,007;
580 $\mathrm{s}^{-1}$ et barres de Hopkinson de l'École polytechnique $\left.[160 ; 730] \mathrm{s}^{-1}\right)$. La gamme de vitesse de déformation atteinte par la traction indirecte est comprise entre 200 et environ $450 \mathrm{~s}^{-1}$ pour des longueurs utiles d'éprouvettes de $11,3 \mathrm{~mm}$ et de section unitaire $4,7 \mathrm{~mm}^{2}$. Les bornes du domaine de recouvrement aux vitesses de déformation dites « intermédiaires » ont ainsi été entrevues sur la base des spécificités de chacun des deux moyens d'essais et de mesure utilisés ici.

Remerciements. Les auteurs tiennent à remercier la participation du Professeur G. Gary - Directeur de Recherche au Laboratoire de Mécanique des Solides de l'École polytechnique de Palaiseau - pour la mise à disposition du montage de traction sur éprouvettes pliées et sa contribution pour la mise en ouvre $\mathrm{du}$ protocole de calibration de notre dispositif d'essais de traction aux barres de Hopkinson implanté sur le site de Lille de l'Onera, A. Deudon pour sa contribution dans la réalisation des essais sur vérin hydraulique rapide et $\mathrm{P}$. Mahelle pour son aide dans la mise en œuvre et la réalisation des essais au moyen du montage de traction par chargement indirect sur éprouvettes plates collées.

\section{Références}

[1] R.O. Fehr, E.R. Parker, Measurement of dynamic stress and strain in tensile test specimens, Proceedings of the Society for Experimental Stress Analysis 1 (1943) 76-82

[2] R.M. Davies, A critical study of the Hopkinson pressure bar, Phil. Trans. R. Soc. Lon. A 240 (1948) 375-457

[3] R.F. Steidel, C.E. Makerov, The tensile properties of some engineering materials at moderate rates of strain, ASTM Bulletin 247 (1960) 57-64

[4] J.E. Smith, Tension tests of metals at strain rates up to $200 \mathrm{~s}^{-1}$, Materials Research and Standards, 1963, pp. $713-718$

[5] P. Béguelin, Approche expérimentale du comportement mécanique des polymères en sollicitation rapide, Thèse, École polytechnique Fédérale de Lausanne, 1996

[6] J. Fabis, Caractérisation expérimentale de la loi de comportement d'un acier en dynamique, Rapport Onera-Lille $\mathrm{n}^{\circ} 97 / 63,1997$

[7] U.S. Lindholm, R.L. Bessey, G.V. Smith, Effect of strain rate on yield strength, tensile strength, and elongation of three aluminum alloys, J. Mat. 6 (1971) 119-133

[8] T. Nicholas, Dynamic tensile testing of structural materials using a split Hopkinson bar apparatus, Technical report, AFWAL TR 80-4053, 1980

[9] I.H. Hove, B. Andersson, T.E. Johnsen, High speed tensile testing, J. Physique IV, C3, 1997, pp. 229-234

[10] A. Mansilla, A. Regidor, D. Garcia, A. Negro, Dynamic tensile testing for determining the stress-strain curve at different strain rate, J. Physique IV 10 (2000) 695-700

[11] M. Quik, K. Labibes, C. Albertini, T. Valentin, P. Magain, Dynamic mechanical properties of automotive thin sheet steel in tension, compression and shear, J. Physique IV, Colloque C3, Supplément au J. Physique III 24 (1997) 379-384

[12] J. Fabis, Contribution à la caractérisation en dynamique rapide de lois de comportement de matériaux composites, Rapport Onera-Lille n ${ }^{\circ}$ 99/40, 1999 
[13] J. Rodriguez, C. Navarro, V. Sanchez $\sim$ Galvez, Numerical assessment of the dynamic tension test using the Split Hopkinson Bar, J. Testing and Evaluation, nº 422 (1994) 335-342

[14] A. Rusinek, J.R. Klepaczko, Comportement viscoplastique des tôles en traction et cisaillement - Analyse de la vitesse d'impact, Matériaux \& Techniques 11-12 (1999) 41-52

[15] H. Zhao, Analyse de l'essai aux barres d'Hopkinson Application à la mesure du comportement dynamique des matériaux, Thèse, École Nationale des Ponts et Chaussées, 1992

[16] L. Rota, Application de méthodes inverses au dépouillement de l'essai aux barres de Hopkinson, Thèse, École polytechnique, 1997

[17] H. Kolsky, Stress waves in solids, Dover Publications Inc., New York, 1963

[18] G. Gary, Some aspects of dynamic testing with waveguides, New experimental Methods in Material Dynamics and Impact, Warsaw 1, Poland, 2001, pp. 179-222

[19] Y. Sato, H. Takeyama, The use of the split Hopkinson pressure bar to obtain dynamic stress/strain data at constant strain rates, Technology Reports, Tohoku University, n 243 (1978) 303-315

[20] U.S. Lindholm, L.M. Yeakley, Some experiments with the split Hopkinson pressure bar, Experimental Mechanics 12 (1964) 317-355

[21] DYMAT, Présentation des essais dynamiques par barres d'Hopkinson, Document DYMAT ENSTA/DYMAT M10

[22] P. Mouro, G. Gary, H. Zhao, Dynamic tensile testing of sheet metal, J. Physique IV 10 (2000) 149-154

[23] G. Gary, J.R. Klepaczko, A computer program for the analysis of the split Hopkinson bars test, Paris/Metz, 1988

[24] G. Gary, J.R. Klepaczko, H. Zhao, Correction de dispersions pour l'analyse des petites déformations aux barres de Hopkinson, J. Physique IV 1 (1991) 403-410

[25] M.M. Leblanc, D.H. Lassila, Dynamic tensile testing of sheet material using the Split Hopkinson Bar technique, Experimental Techniques 17 (1993) 37-42

[26] T. Yokoyama, T. Isomoto, Impact tension testing of sheet metals for automobile structural uses, Proceedings of Asian Pacific Conference for Fracture and Strength, 1996, 795-799

[27] C.H. Nguyen, H.J. Schindler, On spurious reflection waves in Hopkinson bar tensile tests using a collar, J. Physique IV 7 (1997) 85-90

[28] J. Harding, E.O. Wood, J.D. Campbell, Tensile testing of materials at impact rates of strain, J. Mechanical Engineering Science 2 (1960) 88-96
[29] J.M. Yuan, V.P.W. Shim, Tensile response of ductile $\alpha-$ titanium at moderately high strain rates, Int. J. Solids and Structures 39 (2002) 213-224

[30] I.S. Chocron-Benloulo J. Rodriguez, M.A. Martinez, V. Sanchez Galvez, Dynamic tensile testing of aramid and polyethylene fiber composites, Int. J. Impact Engineering 19 (1997) 135-146

[31] C. Kammerer, Modélisation du comportement plan d'un composite quasi-unidirectionnel en verre $\mathrm{E} /$ polyester sous faibles et fortes vitesses de déformation - Application au cas de l'impact, Thèse, Université de Paris XIII, 1996

[32] D.D. Radford, M.J. Worswick, The mechanical and constitutive behaviour of as-received and irradiated $\mathrm{Zr}$ $2.5 \mathrm{Nb}$ pressure tube material under high rates of tensile strain, J. Physique IV (2000) 293-298

[33] M.A. Kaiser, Advancements in the split Hopkinson bar test, Thesis of Mechanical Engineering, Faculty of the Virginia Polytechnic Institute, 1998

[34] H. Eskandari, J.A. Nemes, Dynamic testing of composite laminates with a tensile split Hopkinson bar, J. Composites Materials 34 (2000) 260-273

[35] B.P. Zhang, C. Ding, B. Liu, H. Lin, S.H. Zhou, Dynamic tensile behavior of $93 \mathrm{Wt} \%$ tungsten alloy and its fractal features of fracture, J. Physique IV C3 (1997) 409-414

[36] G. Haugou, J. Fabis, B. Langrand, E. Deletombe, E. Markiewicz, Iterative experimental/numerical procedure for improvement of dynamic experimental facilities, Conference of Structures under Shock and Impact, Montreal, Qc, Canada, 2002, pp. 113-122

[37] H. Zhao, G. Gary, The testing and behaviour modelling of sheet metals at strain rates from $10^{-4}$ to $10^{4} \mathrm{~s}^{-1}$, Materials Science and Engineering 207 (1996) $46-50$

[38] G. Gary, H. Zhao, Étude expérimentale du comportement dynamique des matériaux, Mécanique \& Industries 1 (2000) 15-26

[39] G. Haugou, Moyens d'essais et de caractérisation de lois de comportement matérielles en dynamique moyennes vitesses, Thèse, Université de Valenciennes, 2003

[40] M.N. Bussac, P. Collet, G. Gary, R. Othman, An optimisation method for separating and rebuilding onedimensional dispersive waves from multi-point measurements - Application to elastic or viscoelastic bars, J. Mechanics and Physics of Solids 50 (2002) 321-350

[41] R. Othman, Extension du champ d'application du système des barres de Hopkinson aux essais à moyennes vitesses de déformation, Thèse, École polytechnique, 2002 